\title{
CORRESPONDENCE
}

\section{Limiting the areas inspected by lung ultrasound leads to an underestimation of COVID-19 patients' condition}

\author{
Federico Mento ${ }^{1}$ (10, Tiziano Perrone ${ }^{2} \mathbb{B}$, Anna Fiengo ${ }^{2}$, Francesco Tursi ${ }^{3}$, Veronica Narvena Macioce ${ }^{3}$, \\ Andrea Smargiassi ${ }^{4}$ (D) Riccardo Inchingolo ${ }^{4}$ and Libertario Demi $i^{*^{*}}$
}

@ 2021 Springer-Verlag GmbH Germany, part of Springer Nature

We read with interest the work by Volpicelli and colleagues [1]. We strongly agree on the usefulness of lung ultrasound (LUS) in the management of patients affected by coronavirus disease 2019 (COVID-19), and share the authors' view concerning the role of LUS, which is not to diagnose the infection but to detect the presence of associated interstitial pneumonia and monitor its evolution [2]. We also consider it very important to highlight once more the non-specificity of LUS patterns for COVID-19 pneumonia.

We are, however, interested in understanding the rationale behind the authors' choice of limiting the inspected areas to six.

In a recent work, we compared the impact of different imaging protocols in the evaluation of COVID-19 patients by LUS and found that a minimum of 10 areas was necessary to avoid a significant underestimation of the patient's condition [3].

We have now looked at a broader patients' population, analyzed in detail the implication of a six-area inspection, and compared it with our proposed fourteen-area approach [4].

This multicenter study was part of a registered protocol (NCT04322487) and received approval from the Ethical Committee of the Fondazione Policlinico Universitario Agostino Gemelli, Istituto di Ricovero e Cura a Carattere Scientifico (protocol 0015884/20 ID 3117), of Milano area 1, the Azienda Socio-Sanitaria Territoriale

*Correspondence: libertario.demi@unitn.it

${ }^{1}$ Department of Information Engineering and Computer Science, University of Trento, Trento, Italy

Full author information is available at the end of the article
Fatebenefratelli-Sacco (protocol N0031981), and the Fondazione Policlinico Universitario San Matteo (protocol 20200063198).

All patients gave informed consent.

In total, 1807 LUS videos from 100 patients (59 males and 41 females, with a mean age of 61.7), corresponding to 367,263 LUS frames, were collected and analyzed. All patients were COVID-19 positive, confirmed by reverse transcription-polymerase chain reaction test. LUS data have been acquired using a convex probe, setting an imaging depth from 8 to $12 \mathrm{~cm}$ (depending on the patient) and an imaging frequency from 3.5 to $6.6 \mathrm{MHz}$ (depending on the scanner).

As from [1] it was not possible to retrieve an unambiguous positioning of the posterior areas, we compared the findings from our fourteen-area protocol with three six-area protocols. The left and right anterior-apical (area 12 and 14) and lateral-apical (8 and 10) areas were always included,while for the posterior area, we include only the left and right apical (6 and 3), medial (5 and 2), or basal areas (4 and 1). These are referred as protocols 1, 2, and 3, respectively (Fig. 1).

Results show a 43, 63, and $80 \%$ agreement with our fourteen-area protocol for protocols 1,2 and 3, respectively, when inspecting the data for bilateral large consolidations and/or areas of white lung (large hyperechoic bands). Moreover, a 77, 87, and $87 \%$ agreement was found for protocols 1, 2, and 3, respectively, when inspecting the data for bilateral small or large consolidations and/or areas of white lung. These results would imply an underestimation, according to the classification presented in [1], of HighLUS patients as IntLUS patients. Moreover, as previously reported in [3], these results confirm the

\section{Springer}




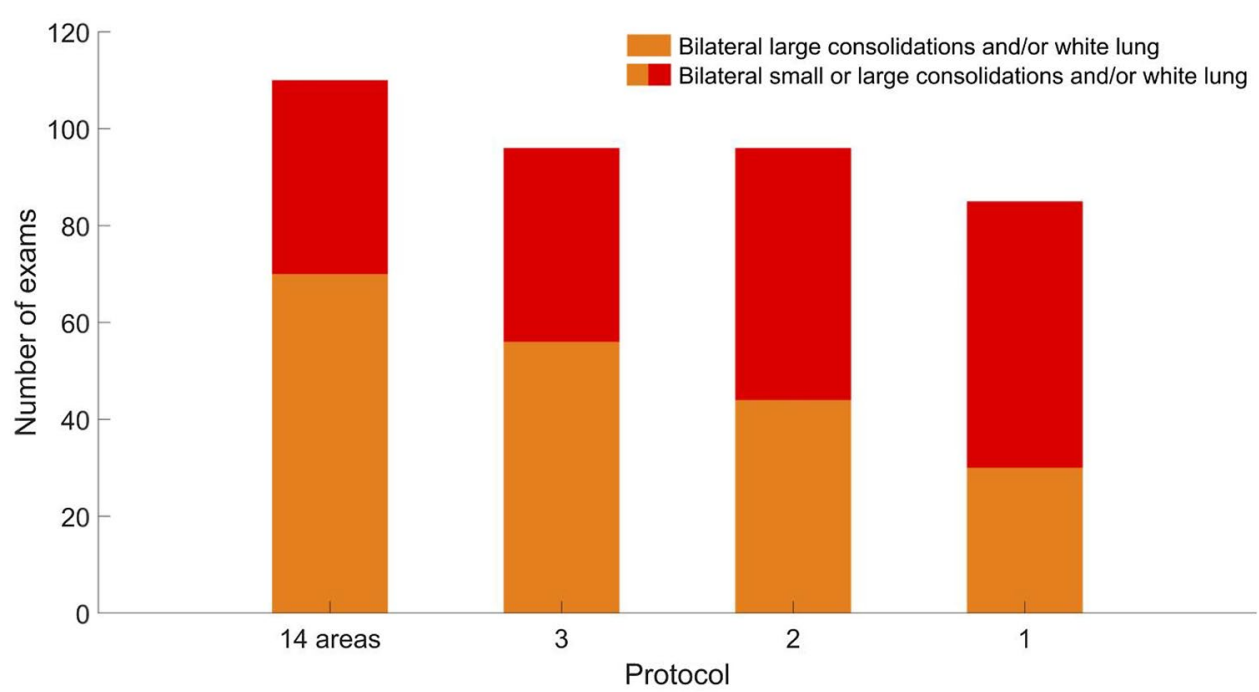

Fig. 1 For each protocol, the number of exams with bilateral large consolidations and/or white lung is represented as orange bars. The combination of orange and red bars represents the number of exams with bilateral small or large consolidations and/or white lung. Here, we refer to exams rather than patients as some patients were examined multiple times (on different dates), obtaining a total number of exams of 133 rather than 100. Of the 133 exams, 110 exams had bilateral small or large consolidations and/or white lung

importance of the basal areas for a correct evaluation of LUS data from COVID-19 patients.

\section{Author details}

${ }_{1}^{1}$ Department of Information Engineering and Computer Science, University of Trento, Trento, Italy. ${ }^{2}$ Department of Internal Medicine, IRCCS San Matteo, Pavia, Italy. ${ }^{3}$ UOS Pneumologia Di Codogno, Asst Lodi, Lodi, Italy. ${ }^{4}$ Pulmonary Medicine Unit, Department of Medical and Surgical Sciences, Fondazione Policlinico Universitario A. Gemelli IRCCS, Rome, Italy.

\section{Funding}

This work was supported by the European Institute of Technology (project UltraOn, EIT Digital 2020) and the Fondazione Valorizzazione Ricerca Trentina (grant 1, COVID-19 2020).

\section{Declarations}

\section{Conflict of interest}

The authors declare no conflicts of interest.

\section{Publisher's Note}

Springer Nature remains neutral with regard to jurisdictional claims in published maps and institutional affiliations.
Received: 2 April 2021 Accepted: 7 April 2021 Published online: 11 May 2021

\section{References}

1. Volpicelli G, Gargani L, Perlini S et al (2021) Lung ultrasound for the early diagnosis of COVID-19 pneumonia: an international multicenter study. Intensive Care Med. https://doi.org/10.1007/s00134-021-06373-7

2. Perrone T, Soldati G, Padovini L et al (2020) A New lung ultrasound protocol able to predict worsening in patients affected by severe acute respiratory syndrome Coronavirus 2 pneumonia. J Ultrasound Med. https://doi. org/10.1002/jum.15548

3. Mento F, Perrone T, Macioce VN et al (2020) On the impact of different lung ultrasound imaging protocols in the evaluation of patients affected by Coronavirus disease 2019: how many acquisitions are needed. J Ultrasound Med. https://doi.org/10.1002/jum.15580

4. Soldati G, Smargiassi A, Inchingolo R et al (2020) Proposal for international standardization of the use of lung ultrasound for COVID-19 patients; a simple, quantitative, reproducible method. J Ultrasound Med 39(7):14131419. https://doi.org/10.1002/jum.15285 